\title{
Essential role of DOT1L in maintaining normal adult hematopoiesis
}

Cell Research (2011) 21:1370-1373. doi:10.1038/cr.2011.115; published online 19 July 2011

\section{Dear Editor,}

Histone methylation plays important roles in regulating gene expression and diverse biological processes [1]. Methylation of a unique residue, lysine 79 of histone $\mathrm{H} 3$ (H3K79), is evolutionarily conserved from yeast to human and is catalyzed by yeast Dot1 (disruptor of telomeric silencing) and its mammalian homolog DOT1L (Dot1-Like), respectively [2]. Previous studies have revealed that Dot1/DOT1L and its associated H3K79 methylation mark are involved in various biological processes including transcriptional regulation, DNA repair, and cell cycle regulation [3]. In addition, DOT1L and H3K79 methylation are also important for cardiac function [4] and leukemogenesis [5-7]. The fact that DOT1L, particularly its enzymatic activity, is involved in multiple MLL-related acute leukemias implies its potential role as a therapeutic target. However, the role of DOT1L in normal hematopoiesis is still unknown. Such information is critical for developing a DOT1L-based therapeutic strategy against acute leukemia. Here we use DOT1Lknockout mice coupled with bone marrow transplantation to demonstrate that DOT1L plays an important role in adult hematopoiesis.

Since germ-line DOT1L-knockout mice die before definitive hematopoiesis at embryonic stage [8], we generated an inducible DOT1L-knockout mouse line to evaluate the role of DOT1L in adult hematopoiesis. To this end, we crossed the DOT1L conditional mice with a Cre-ER mouse line (R26-Cre-ER), so that DOT1L can be deleted in vivo by Tamoxifen (TAM) injection (Supplementary information, Data $\mathrm{S} 1$ ). To examine the role of DOT1L in normal adult hematopoiesis, 8-12-weekold wild-type (DOT1L $\left.\mathrm{L}^{\mathrm{w} / \mathrm{wt}} / \mathrm{Cre}-\mathrm{ER}\right)$ and heterozygous (DOT1L $L^{2 \text { lox/10x }} /$ Cre-ER) mice were administered TAM every 2 days for a period of 2 weeks. RT-qPCR analysis demonstrated that this regimen of TAM treatment can induce efficient recombination leading to DOT1L depletion (Supplementary information, Figure S1). One week after the last injection, the DOT1 $\mathrm{L}^{2 \mathrm{lox} / 10 x} / \mathrm{Cre}-\mathrm{ER}$ mice began to display signs of anemia and bleeding, while the wild- type mice appeared normal. Pathological examination demonstrated that the DOT1 $1 \mathrm{~L}^{2 \operatorname{lox} / 10 x} / \mathrm{Cre}-\mathrm{ER}$ mice display severe anemia in internal organs such as the liver (Figure 1A). In addition, brain hemorrhaging is observed upon loss of DOT1L in the DOT1L $\mathrm{L}^{210 \mathrm{x} / \mathrm{lox}} / \mathrm{Cre}-\mathrm{ER}$ mice, which is absent in the DOT1L ${ }^{\mathrm{wt} / \mathrm{wt}} / \mathrm{Cre}-\mathrm{ER}$ controls (Figure 1B). Consistent with these observations, a general hypocellularity in the bone marrow of DOT1 $1 \mathrm{~L}^{2 \mathrm{lox} / 10 x} / \mathrm{Cre}-\mathrm{ER}$ mice was also observed when compared to wild-type controls (Figure 1C).

To determine the effect of DOT1L depletion in hematopoietic lineage development, FACS analyses were performed on bone marrow cells isolated from the experimental mice (Supplementary information, Data $\mathrm{S} 1$ ). The results demonstrated that the percentages of multiple progenitors including CMP (common myeloid progenitors), GMP (granulocyte/monocyte progenitors), and MEP (megakaryocyte/erythrocyte progenitors) are reduced significantly in DOT1L $\mathrm{L}^{2 \mathrm{lox} / \mathrm{lox}} / \mathrm{Cre}-\mathrm{ER}$ mice compared with the wild-type control (Figure 1D, Supplementary information, Figure S2). Consistently, terminally differentiated myeloid lineage cells (Gr-1+ or Mac-1+) are also significantly reduced upon DOT1L deletion (Figure 1E, Supplementary information, Figure S2). Collectively, these data indicate that DOT1L is critical in maintaining normal adult hematopoiesis and that depletion of DOT1L leads to bone marrow failure.

To determine whether Dot1L deletion-induced bone marrow failure observed above is caused by autonomous defects of hematopoietic cells, we performed bone marrow transplantation in which the DOT1 $1 \mathrm{~L}^{2 \mathrm{lox} / 10 \mathrm{x}} / \mathrm{Cre}-\mathrm{ER}$ or DOT1L $\mathrm{L}^{\mathrm{wt} / \mathrm{wt}} / \mathrm{Cre}-\mathrm{ER}$ donor cells were transplanted into congenic recipient mice (Supplementary information, Data S1). Four weeks after bone marrow reconstitution, TAM was administrated for 2 weeks to delete DOT1L in DOT1L $\mathrm{L}^{210 x / 10 x} / \mathrm{Cre}-\mathrm{ER}$ donor cells. Two weeks after the last TAM injection, total bone marrow cells were isolated and counted. Compared to mice transplanted with wildtype donor cells, total bone marrow cells in the recipient mice transplanted with DOT1 $\mathrm{L}^{210 x / 10 x} /$ Cre-ER cells are significantly reduced (Figure $1 \mathrm{~F}$ ). This result suggests 
A

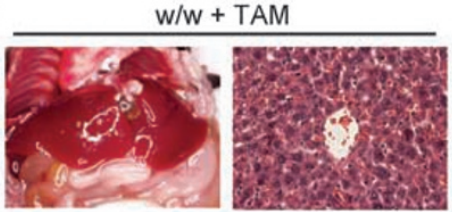

2lox/1lox + TAM

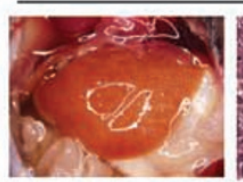

C

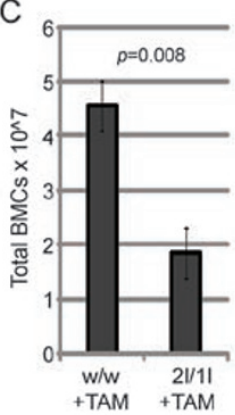

B

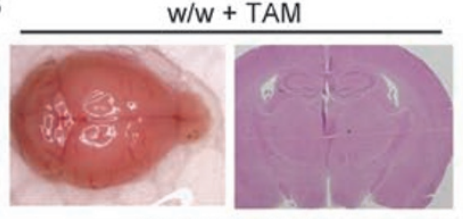

2lox/1lox + TAM

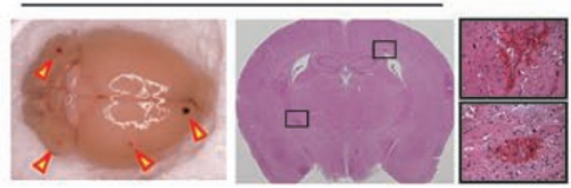

E 70

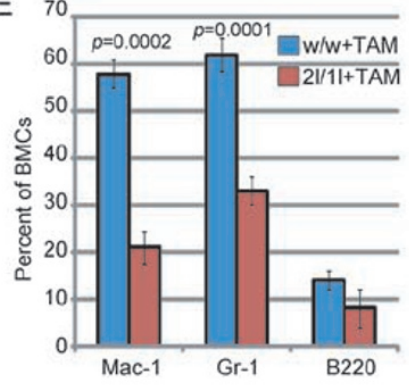

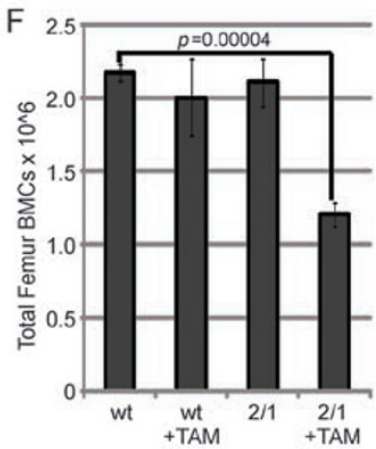

Ly5.2 ㄴy5.1(Competitor)

G

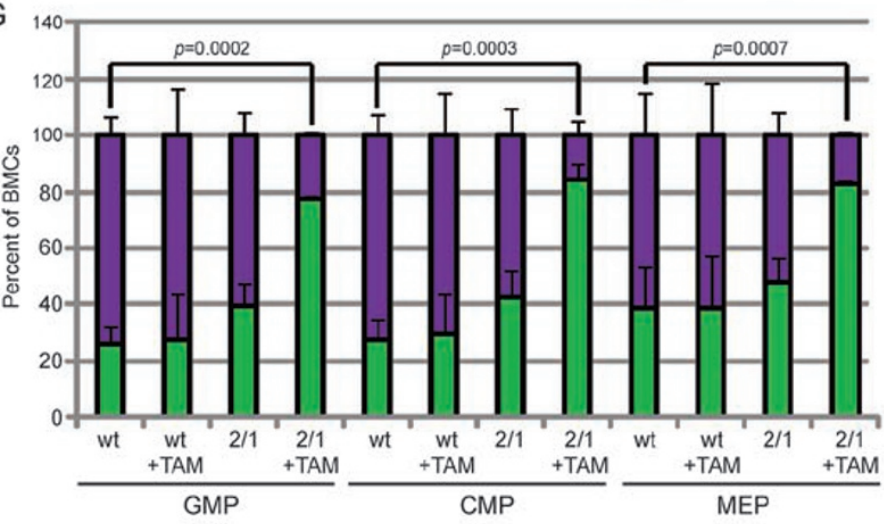

D

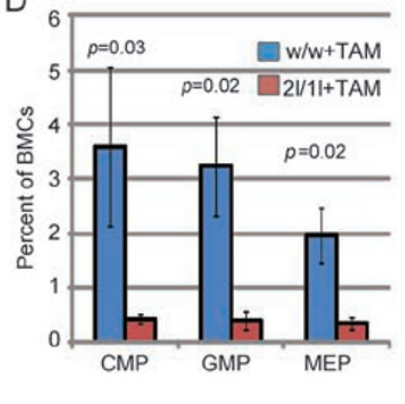

H ${ }_{140}$

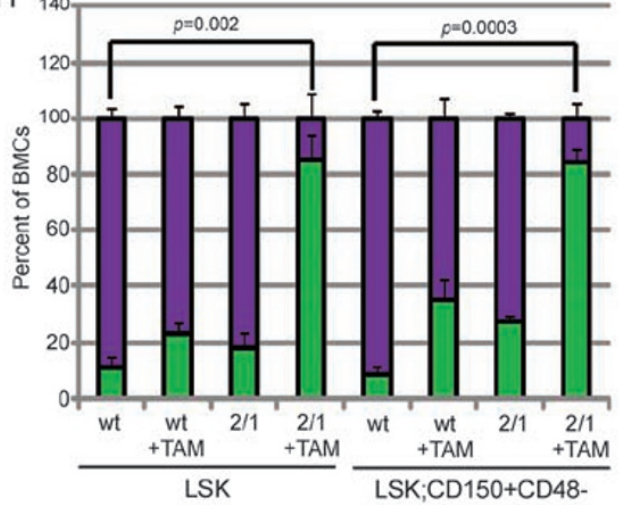

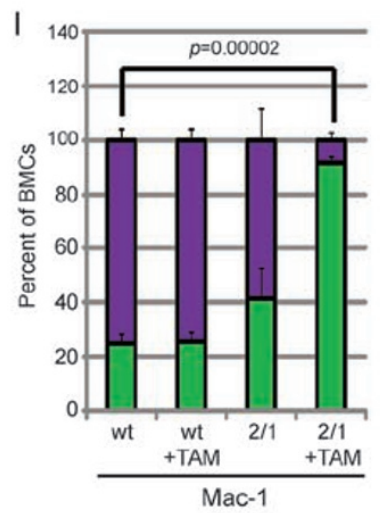

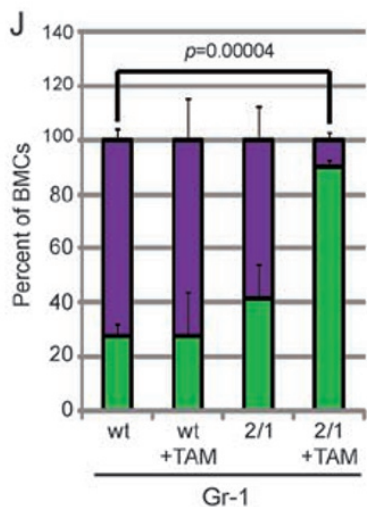

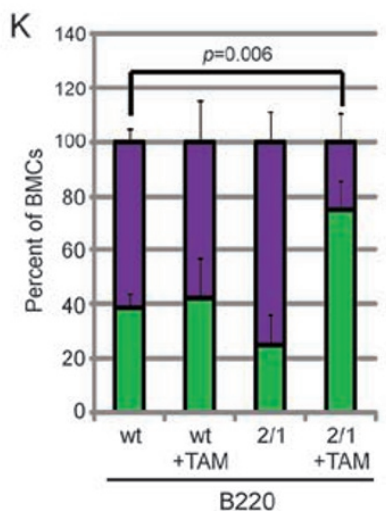

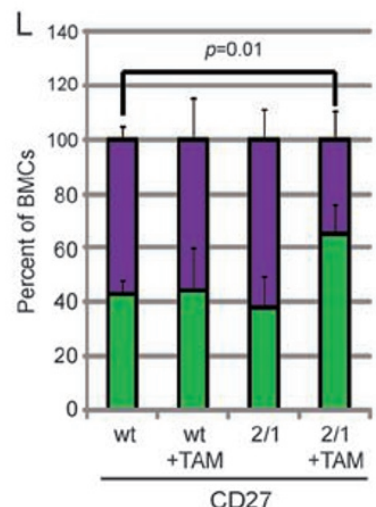

a competition assay in which Ly5.1+ wild-type cells were mixed with Ly5.2+ DOT1L $\mathrm{L}^{2 \mathrm{lox} / 10 \mathrm{x}} / \mathrm{Cre}-\mathrm{ER}$ or Ly5.2+ DOT1L $L^{\mathrm{wt} / \mathrm{wt}} /$ Cre-ER donor cells $(1: 1$ ratio) and trans- 
Figure 1 DOT1L enzymatic activity is required for normal hematopoiesis. (A) TAM-induced deletion of DOT1L in adult mice (2lox/1lox + TAM) results in anemia as shown by pale livers and absence of red blood cells in liver tissue sections stained with H\&E ( $n=3$ mice) compared to w/w + TAM controls. DOT1L ${ }^{+/+} /$Cre-ER mice are labeled as w/w and DOT1L $\mathrm{L}^{2 / 0 x / 110 x} / \mathrm{Cre}-$ ER mice are labeled as 2lox/1lox. (B) TAM-induced deletion of DOT1L (2lox/1lox + TAM) results in brain hemorrhage (red arrows), which was not observed in TAM-treated w/w controls. Magnified images of representative hemorrhages from H\&E stained tissue sections are shown $(n=3$ mice). (C) Total bone marrow cell (BMC) count from femur and tibia aspirates demonstrates hypocellularity in DOT1L-deleted mice (2l/1I+TAM) compared to their controls (w/w+ TAM) ( $n=3$ mice). (D) FACS analysis of BMCs revealed that TAM-induced DOT1L deletion (2I/1I +TAM) results in decreased percentage of CMP, GMP, and MEP lineage cells when compared to those from the control mice ( $n=3$ mice). (E) FACS analysis of BMCs shows that the percentage of differentiated cells - macrophages (Mac-1), granulocytes (Gr-1), and B-cells (B220) - are significantly reduced in response to DOT1L deletion (2I/1I + TAM). ( $n=3$ mice). (F) Bone marrow transplantation followed by total BMC count demonstrates that the hypocellularity effect upon DOT1L loss of function (2/1+TAM) is cell autonomous. Bone marrow competition assays were performed by co-transplanting wild-type Ly5.1 Cre-negative cells (competitor) with DOT1L $\mathrm{L}^{+/+} / \mathrm{Cre}$-ER Ly5.2 cells (wt) or DOT1L $210 x / 110 x / C r e-E R$ Ly5.2 cells (2/1) into wild-type Ly5.1 mice. (G) FACS analysis indicates that deletion of DOT1L results in cell autonomous loss of progenitor cells as shown by a reduced percentage of Ly5.2 cells in TAM-treated mice transplanted with DOT1L ${ }^{210 x / 110 x} /$ Cre-ER Ly5.2 cells (2/1+TAM). (H) FACS analysis indicates that DOT1L deletion results in cell autonomous loss of LSK (Lin-/Sca1+/cKit+) hematopoietic stem cells (HSCs) as well as long-term reconstituting HSCs (Lin-/Sca1+/cKit+/CD150+/CD48-), as shown by a reduced percentage of Ly5.2 cells in TAM-treated mice transplanted with DOT1L ${ }^{2 / 0 x / 110 x} /$ Cre-ER Ly5.2 cells (2/1+ AM). (I-L) FACS analysis indicates that deletion of DOT1L results in cell autonomous loss of differentiated cells (Mac-1, Gr-1, B220, and CD27) as shown by a reduced percentage of Ly5.2 cells in TAM-treated mice transplanted with DOT1L ${ }^{2 / 0 x / 110 x} /$ Cre-ER Ly5.2 cells (2/1 + TAM).

planted into congenic mice. Four weeks after bone marrow reconstitution, TAM was administered for 2 weeks to deplete DOT1L in DOT1 $\mathrm{L}^{2 \operatorname{lox} / 10 x} / \mathrm{Cre}-\mathrm{ER}$ donor cells. The contribution of wild-type (Ly5.1+) and DOT1Ldepleted donor cells (Ly5.2+) to different hematopoietic lineages was then monitored by the percent change of Ly5.1+/Ly5.2+ cells. This analysis demonstrated that after TAM-induced DOT1L depletion, the percentages of Ly5.2+ DOT1L $\mathrm{L}^{2 \mathrm{lox} / 10 \mathrm{x}} / \mathrm{Cre}$-ER donor cells are significantly decreased in CMP, GMP, and MEP populations (Figure 1G, Supplementary information, Figure S3). We also observed significant decrease in the percent contribution of DOT1L-depleted Ly5.2+ cells in LSK (Lin-/ $\mathrm{Sca} 1+/ \mathrm{cKit}+$ ) hematopoietic stem cells (HSCs) as well as long-term reconstituting HSCs (Lin-/Sca1+/cKit+/ CD150+/CD48-) population (Figure 1H, Supplementary information, Figure S4). Finally, the percentage of DOT1L-depleted Ly5.2+ donor cells was also decreased in terminally differentiated myeloid (Gr-1+/Mac-1+) and lymphoid (B220+/CD27+) populations (Figure 1I-1L, Supplementary information, Figures S5 and S6). Collectively, results from the bone marrow transplantation experiments support our conclusion that DOT1L is critical for maintaining normal adult hematopoiesis in mice.

In this study, we demonstrated that DOT1L plays an important role in maintaining normal adult hematopoiesis using conditional DOT1L-knockout mice coupled with bone marrow transplantation assay. Knockout of DOT1L in bone marrow cells depletes hematopoietic stem cells, various progenitor cell populations, as well as terminally differentiated blood cell lineages supporting a role for
DOT1L in maintaining the hematopoietic progenitor and stem cell population. Using a similar approach, a recent study from the Hess group also reached the same conclusion [9]. Interestingly, DOT1L is also required for embryonic erythropoiesis [10]. Although the demonstration that DOT1L plays an important role in adult hematopoiesis raises concerns for targeting DOT1L for leukemia treatment, it does not exclude the possibility of using small molecule inhibitors that target DOT1L since it is possible that normal hematopoietic stem/progenitors and leukemic cells may have different sensitivities to DOT1L inhibition. Therefore, comparing the sensitivity of normal hematopoietic stem/progenitor cells with leukemia cells to potent DOT1L inhibitors will be important in determining the therapeutic window.

\section{Acknowledgments}

We thank Susan Wu (UNC-CH) for critical reading of the manuscript. The work is supported by an NIH grant (CA119133). A T N was a recipient of the Pre-doctoral Fellowship from the American Heart Association. Y Z is an Investigator of the Howard Hughes Medical Institute.

Anh T Nguyen ${ }^{1,2, *}$, Jin $\mathrm{He}^{1,2, *}$, Olena Taranova ${ }^{1,2}$, Yi Zhang,

${ }^{1}$ Howard Hughes Medical Institute, ${ }^{2}$ Department of Biochemistry and Biophysics, Lineberger Comprehensive Cancer Center, University of North Carolina at Chapel Hill, Chapel Hill, NC27599-7295, USA 
*These two authors contributed equally to this work. Correspondence: Yi Zhang

Tel: +919-843-8225; Fax: +919-966-4330

E-mail: yi_zhang@med.unc.edu

\section{References}

1 Martin C, Zhang Y. The diverse functions of histone lysine methylation. Nat Rev Mol Cell Biol 2005; 6:838-849.

2 Feng Q, Wang H, Ng HH, et al. Methylation of H3-lysine 79 is mediated by a new family of HMTases without a SET domain. Curr Biol 2002; 12:1052-1058.

3 Nguyen AT, Zhang Y. The diverse functions of DOT1 and H3K79 methylation. Genes Dev 2011; 25:1345-1358.

4 Nguyen AT, Xiao B, Neppl RL, et al. DOT1L regulates dystrophin expression and is critical for cardiac function. Genes Dev 2011; 25:263-274.

5 Krivtsov AV, Feng Z, Lemieux ME, et al. H3K79 methylation profiles define murine and human MLL-AF4 leukemias. Cancer Cell 2008; 14:355-368.

6 Mueller D, Bach C, Zeisig D, et al. A role for the MLL fusion partner ENL in transcriptional elongation and chromatin modification. Blood 2007; 110:4445-4454.

7 Okada Y, Feng Q, Lin Y, et al. hDOT1L links histone methylation to leukemogenesis. Cell 2005; 121:167-178.

8 Jones B, Su H, Bhat A, et al. The histone H3K79 methyltransferase Dot1L is essential for mammalian development and heterochromatin structure. PLoS Genet 2008; 4:e1000190.

9 Jo SY, Granowicz EM, Maillard I, Thomas D, Hess JL. Requirement for Dot11 in murine postnatal hematopoiesis and leukemogenesis by MLL translocation. Blood 2011; 117:47594768.

10 Feng Y, Yang Y, Ortega MM, et al. Early mammalian erythropoiesis requires the Dot1L methyltransferase. Blood 2010; 116:4483-4491.

(Supplementary information is linked to the online version of the paper on the Cell Research website.) 